\title{
EVIDÊNCIAS DA FORMAÇÃo DE MONOCAMADA DE ÓXIDO DE ALUMÍNIO SOBRE SÍLICA, ATRAVÉS DE REAÇÕES DE ENXERTO
}

Julia M. D. Cónsul, Ione M. Baibich, Edilson V. Benvenutti* e Daniel Thiele

Instituto de Química, Universidade Federal do Rio Grande do Sul, CP 15003, 91501-970 Porto Alegre - RS

Recebido em 14/1/04; aceito em 15/10/04; publicado na web em 2/2/05

\begin{abstract}
EVIDENCE OF ALUMINUM OXIDE MONOLAYER FORMATION ON A SILICA GEL SURFACE USING GRAFTING REACTIONS. Aluminum oxide was dispersed on a commercial silica gel surface, using successive grafting reactions. The reaction products were characterized by $\mathrm{N}_{2}$ adsorption-desorption isotherms, scanning electron microscopy and infrared spectroscopy. The progressive incorporation of aluminum, up to $5.5 \%(\mathrm{w} / \mathrm{w})$, does not produce agglomeration of alumina, since changes in the original pore size distribution of the silica matrix were not observed. The aluminum oxide covers homogeneously the silica surface.
\end{abstract}

Keywords: grafting; atomic dispersion, surface synthesis.

\section{INTRODUÇÃO}

O suporte inorgânico é um aspecto importante a ser considerado no processo de preparação de um catalisador heterogêneo. Além das características físicas como porosidade e rigidez estrutural, o suporte também pode contribuir para a estabilização e prevenção de sinterização das espécies cataliticamente ativas, que se encontram dispersas em sua superfície. Dentre os suportes inorgânicos mais utilizados em catálise heterogênea tem-se a alumina e a sílica ${ }^{1-4}$. As sílicas também têm sido usadas na sua forma modificada. Nesse caso, a modificação química da superfície pode ser feita com outros óxidos inorgânicos ${ }^{3-5}$, ou então, com grupos orgânicos quelantes que podem imobilizar um catalisador metálico na forma de um complexo ${ }^{6,7}$.

Uma das formas de se modificar a superfície de sílicas com outros óxidos inorgânicos é através do uso de reações de enxerto. Com esse método de síntese é possível combinar as características desejáveis das sílicas comercialmente disponíveis, como área superficial, porosidade e granulometria, com a funcionalidade química da superfície dos outros óxidos ${ }^{8-10}$. Portanto, as propriedades físicas originais da matriz sílica não devem ser profundamente alteradas, visto que se admite que as reações de enxerto devem produzir monocamadas de óxido disperso, isto é, filmes bidimensionais dos óxidos enxertados na superfície da sílica.

A modificação química da superfície de sílica pelo método enxerto pode ser descrita, de maneira simplificada, através de duas reações: i) reação dos grupos silanóis da superfície da sílica com o reagente precursor do óxido metálico a ser enxertado, que pode ser um alcóxido ou haleto. Em geral, os alcóxidos são preferidos pois embora menos reativos, não deixam resíduos de haletos no catalisador que possam resultar em efeitos inibidores dos processos catalíticos ${ }^{11}$ e b) reação de hidrólise do alcóxido imobilizado. Para o caso de óxido de alumínio disperso sobre sílica, as reações de enxerto estão representadas pelas Equações 1 e 2.

$$
\begin{aligned}
& \mathrm{n} \equiv \mathrm{SiOH}+\mathrm{Al}(\mathrm{OR})_{3} \rightarrow(\equiv \mathrm{SiO})_{\mathrm{n}} \mathrm{Al}(\mathrm{OR})_{3-\mathrm{n}}+\mathrm{nROH} \\
& (\equiv \mathrm{SiO})_{\mathrm{n}} \mathrm{Al}(\mathrm{OR})_{3-\mathrm{n}}+(3-\mathrm{n}) \mathrm{H}_{2} \mathrm{O} \rightarrow(\equiv \mathrm{SiO})_{\mathrm{n}} \mathrm{Al}(\mathrm{OH})_{3-\mathrm{n}}+(3-\mathrm{n}) \mathrm{ROH}
\end{aligned}
$$

*e-mail: edilson@iq.ufrgs.b onde $\mathrm{R}$ representa um grupo orgânico, geralmente etil, metil ou isopropil.

A modificação da sílica, através de reações de enxerto com vários óxidos inorgânicos, tem sido extensivamente utilizada na obtenção de novos materiais com propriedades adsorventes ${ }^{12,13}$, sensores eletroquímicos ${ }^{14,15}$ e catalisadores ${ }^{4,16,17}$. Em geral, os óxidos de alumínio, titânio e zircônio, enxertados na superfície da sílica, aumentam a acidez da superfície e propiciam melhores resultados em alguns processos catalíticos ${ }^{8,10,18,19}$. Adicionalmente, melhoram as estabilidades química e térmica dos catalisadores ${ }^{3,9.17}$. A presença de óxido de titânio, além de aumentar a acidez da superfície, pode produzir um aumento na densidade eletrônica de catalisadores metálicos dispersos ${ }^{20}$.

Outros métodos de síntese de sílica modificada com óxidos metálicos têm sido propostos como, por exemplo, a deposição física de precursores metálicos, seguida de calcinação ${ }^{18}$. Entretanto, no caso de óxido de alumínio disperso sobre sílica, observou-se a formação de aglomerados de alumina, que resultam em bloqueio parcial dos poros originais da matriz sílica. Esse fechamento dos poros ocorre porque, nas sínteses que envolvem calcinação com reações no estado sólido, a dispersão dos óxidos imobilizados é menor que a obtida pelo método de enxerto.

Entretanto, já foi relatado que o fechamento parcial dos poros também pode ser alcançado a partir de sucessivas reações de enxerto, que resultem em múltiplas camadas de óxido de alumínio disperso. Foi observado que a partir de sucessivas reações de enxerto, de óxido de alumínio sobre sílica, foi possível incorporar até $35 \%$ de alumínio, com significativo fechamento dos poros ${ }^{21}$.

Embora na maioria dos trabalhos publicados sobre o assunto, o método de enxerto seja aceito como um procedimento eficiente na obtenção de filmes finos de óxidos dispersos sobre sílica, pretende-se, neste trabalho, buscar evidências experimentais convincentes para a formação desses filmes finos. Portanto, os objetivos desse trabalho são: i) estudar a evolução química e estrutural de sílica gel comercial, a partir de três sucessivas reações de enxerto de filmes de óxido de alumínio, usando-se quantidades suficientes de precursor isopropóxido de alumínio, para a obtenção de um recobrimento completo da superfície da sílica; ii) verificar se, de fato, é possível obter uma monocamada de óxido de alumínio sobre a superfície de sílica, ou se a alumina formada encontra-se dispersa na forma de aglomerados, que resultem em fechamento parcial 
dos poros originais da matriz sílica. Na caracterização dos materiais usou-se microscopia eletrônica de varredura, isotermas de adsorção e dessorção de nitrogênio e espectroscopia na região do infravermelho.

\section{PARTE EXPERIMENTAL}

\section{Reação de enxerto do óxido de alumínio na superfície de sílica gel}

Reagiu-se 10,00 g de sílica gel (Merck, $250 \mathrm{~m}^{2} \mathrm{~g}^{-1}, 0,2-0,5 \mathrm{~mm}$ ) previamente ativada sob vácuo, durante $8 \mathrm{~h}$ à temperatura de $150{ }^{\circ} \mathrm{C}$, com 1,24 g de isopropóxido de alumínio $\left(\mathrm{C}_{9} \mathrm{H}_{21} \mathrm{AlO}_{3}\right.$, Merck) usando-se $50 \mathrm{~cm}^{3}$ de tolueno seco como solvente. A reação foi mantida sob agitação mecânica por $24 \mathrm{~h}$ a $80{ }^{\circ} \mathrm{C}$, sob atmosfera de argônio. Usando-se funil de vidro sinterizado e vidraria do tipo Schlenk, o produto sólido foi filtrado, sob atmosfera inerte, e lavado sucessivas vezes com tolueno quente, etanol e éter etílico. Posteriormente, o sólido foi seco, sob vácuo a $110^{\circ} \mathrm{C}$, por $4 \mathrm{~h}$. Finalmente foi adicionada água, seguindo-se a secagem do material, sob vácuo a $120^{\circ} \mathrm{C}$, por 4 h. $\mathrm{O}$ sólido resultante foi designado como $\mathrm{Al}_{2} \mathrm{O}_{3} / \mathrm{SiO}_{2}$ (I), considerando-se que o mesmo corresponde à sílica gel modificada com uma primeira camada de óxido de alumínio. Sucessivas camadas de óxido de alumínio foram dispersas na superfície da sílica já modificada com óxido de alumínio, usando-se o mesmo procedimento descrito acima, sendo que os materiais resultantes foram designados de $\mathrm{Al}_{2} \mathrm{O}_{3} / \mathrm{SiO}_{2}$ (II) e $\mathrm{Al}_{2} \mathrm{O}_{3} / \mathrm{SiO}_{2}$ (III), respectivamente para materiais com duas e três camadas de óxido de alumínio.

\section{Microscopia eletrônica de varredura}

Amostras previamente secas em estufa a $110{ }^{\circ} \mathrm{C}$, durante $2 \mathrm{~h}$, foram metalizadas com ouro e submetidas à análise por microscopia eletrônica de varredura e à análise elementar EDS ("Energy Dispersive Spectroscopy"), usando-se o equipamento Jeol, modelo JSM 5800, com uma tensão de $20 \mathrm{KV}$ e aumento de 30000 vezes.

\section{Isotermas de adsorção e dessorção de nitrogênio}

As isotermas de adsorção e dessorção de nitrogênio foram determinadas na temperatura de ebulição do nitrogênio líquido, em um aparelho volumétrico desenvolvido em nosso laboratório, acoplado em um sistema de alto vácuo (Edward turbomolecular). As medidas de pressão foram feitas usando-se um manômetro capilar de $\mathrm{Hg}$. A área superficial das amostras foi determinada utilizandose o método de Brunauer, Emmett e Teller (BET) ${ }^{22}$ e a distribuição do tamanho de poros foi obtida usando-se o método de Barret, Joyner e Halenda $(\mathrm{BJH})^{23}$.

\section{Espectroscopia na região do infravermelho}

Foram preparados discos das amostras dispersas em $\mathrm{KBr}$ seco (0,5\%), com área de $5 \mathrm{~cm}^{2}$ e peso de $c a$. $100 \mathrm{mg}$. Os espectros foram obtidos em um equipamento Shimadzu FTIR, modelo 8300 , com resolução de $4 \mathrm{~cm}^{-1}$ e 100 varreduras.

\section{RESULTADOS E DISCUSSÃO}

Pode-se observar a partir dos resultados da análise elementar, apresentados na Tabela 1, um aumento praticamente linear de incorporação de alumínio nas três sínteses sucessivas. Portanto, a incorporação progressiva de óxido de alumínio na superfície da sílica foi alcançada.
Tabela 1. Percentual mássico obtido por EDS

\begin{tabular}{lccc}
\hline $\mathrm{Amostras}$ & $\mathrm{Si}^{\text {a }}$ & $\mathrm{Al}^{\text {a }}$ & $\mathrm{O}^{\text {a }}$ \\
\hline $\mathrm{Al}_{2} \mathrm{O}_{3} / \mathrm{SiO}_{2}$ (I) & 71,3 & 1,7 & 27,0 \\
$\mathrm{Al}_{2} \mathrm{O}_{3} / \mathrm{SiO}_{2}$ (II) & 65,6 & 3,5 & 30,9 \\
$\mathrm{Al}_{2} \mathrm{O}_{3} / \mathrm{SiO}_{2}$ (III) & 70,9 & 5,5 & 23,7 \\
\hline
\end{tabular}

${ }^{\mathrm{a}}=\% \mathrm{~m} / \mathrm{m}$ com desvio máximo de $10 \%$.

A partir da análise das imagens da microscopia eletrônica de varredura, obtidas com ampliação de 30000 vezes (Figura 1), podese verificar que, tanto a sílica pura como as amostras contendo óxido de alumínio, apresentam superfícies muito semelhantes. Entretanto, pode-se observar um aumento progressivo na uniformidade da superfície com o grau de incorporação de alumínio. A uniformidade na superfície é uma característica desejável, visto que pode significar um aumento nas estabilidades térmica e mecânica do material que poderá ser submetido a condições drásticas de temperatura e fluxo de gases, por exemplo, quando serve de suporte para catalisadores ${ }^{24,25}$. Essas condições são facilmente encontradas em sistemas de escapamento automotivo. A uniformidade dos filmes de alumina dispersos pode torná-los mais resistentes a rupturas e perda de material ativo.
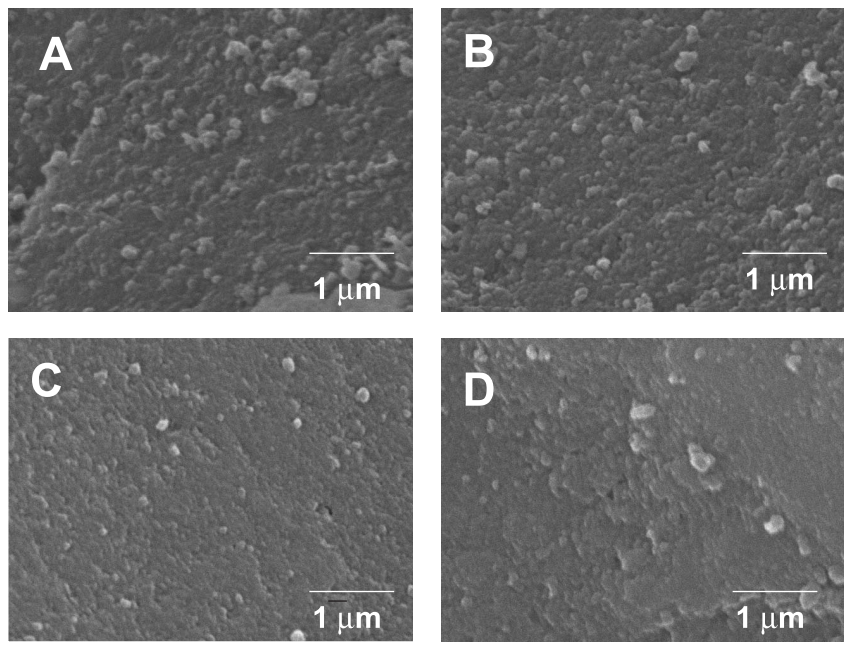

Figura 1. Imagens obtidas por microscopia eletrônica de varredura com ampliação de 30000 vezes. A) sílica pura; $\mathrm{B}) \mathrm{Al}_{2} \mathrm{O}_{3} / \mathrm{SiO}_{2}(\mathrm{I})$; C) $\mathrm{Al}_{2} \mathrm{O}_{3} / \mathrm{SiO}_{2}$ (II) e D) $\mathrm{Al}_{2} \mathrm{O}_{3} / \mathrm{SiO}_{2}(\mathrm{III})$

As isotermas de adsorção e dessorção de nitrogênio dos materiais $\mathrm{Al}_{2} \mathrm{O}_{3} / \mathrm{SiO}_{2}$ (I), $\mathrm{Al}_{2} \mathrm{O}_{3} / \mathrm{SiO}_{2}$ (II) e $\mathrm{Al}_{2} \mathrm{O}_{3} / \mathrm{SiO}_{2}$ (III) são apresentadas na Figura 2, juntamente com a isoterma da sílica pura. Todas as curvas são isotermas típicas do tipo IV, características de materiais mesoporosos ${ }^{26,27}$. Adicionalmente, todas as curvas de dessorção mostram histerese em pressões relativamente altas $\left(\mathrm{P} / \mathrm{P}_{0}>0,5\right)$. Esse comportamento ocorre quando a pressão de equilíbrio na adsorção é maior que a da dessorção. Histereses são muito comuns em materiais mesoporosos e podem ser atribuídas a fatores como condensação capilar de nitrogênio ou estrutura desordenada de poros, que podem ser alongados ou em forma de garrafa ${ }^{26,27}$.

As curvas de distribuição de tamanho dos poros de todas as amostras, incluindo a sílica pura, são mostradas na Figura 3. As amostras, mesmo apresentando diferentes graus de incorporação de óxido de alumínio, apresentam uma mesma distribuição de tamanho dos poros, com máximo em torno de $8 \mathrm{~nm}$. Essa é a mesma distribuição encontrada para a sílica pura. Esse resultado é muito importante, 


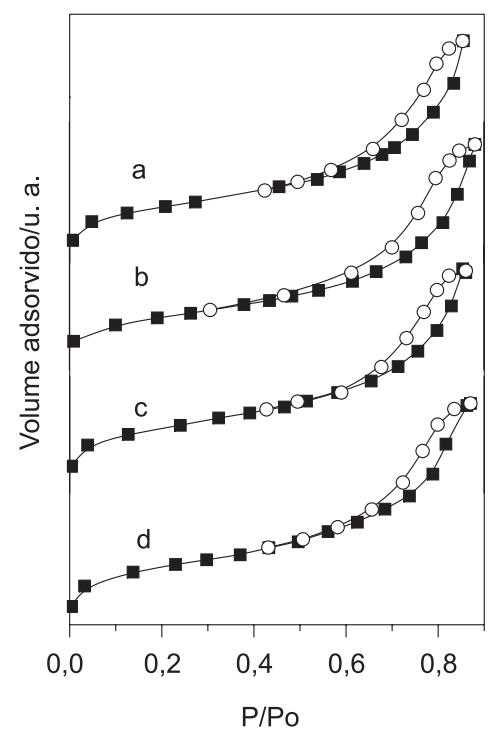

Figura 2. Isotermas de adsorção e dessorção de nitrogênio, obtidas à temperatura de ebulição do nitrogênio. a) sílica pura; b) $\mathrm{Al}_{2} \mathrm{O}_{3} / \mathrm{SiO}_{2}(\mathrm{I})$; c) $\mathrm{Al}_{2} \mathrm{O}_{3} / \mathrm{SiO}_{2}$ (II) e d) $\mathrm{Al}_{2} \mathrm{O}_{3} / \mathrm{SiO}_{2}(\mathrm{III})$

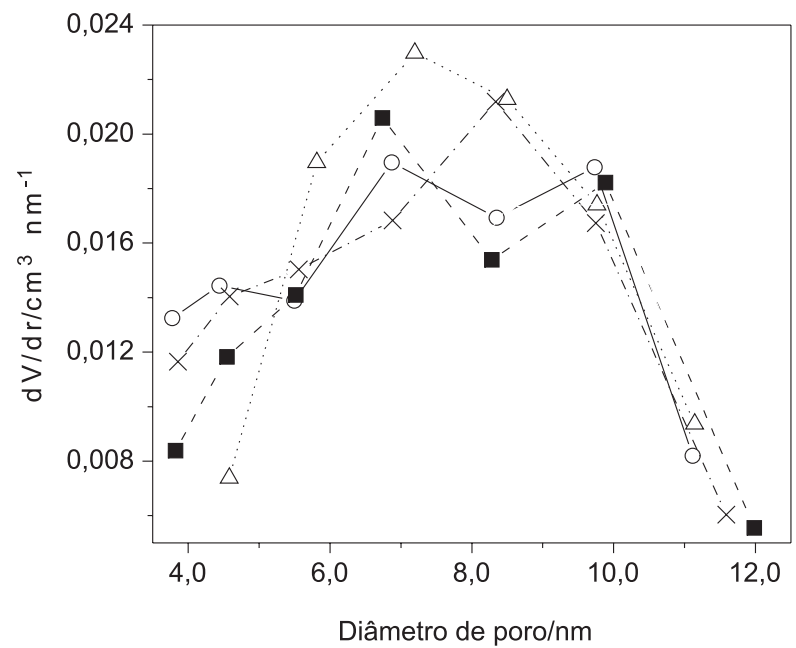

Figura 3. Curvas de distribuição de tamanho dos poros dos materiais. ( $\bigcirc)$ Sílica pura; (ロ) $\mathrm{Al}_{2} \mathrm{O}_{3} / \mathrm{SiO}_{2}(\mathrm{I}) ;(\triangle) \mathrm{Al}_{2} \mathrm{O}_{3} / \mathrm{SiO}_{2}(\mathrm{II})$ e (×) $\mathrm{Al}_{2} \mathrm{O}_{3} / \mathrm{SiO}_{2}(\mathrm{III})$

pois mostra que a incorporação progressiva de alumina na superfície da sílica não produz alterações na sua estrutura de poros original. Esse comportamento somente pode ser explicado considerando-se que a alumina encontra-se, de fato, dispersa na forma de um filme bidimensional (monocamada). A espessura do filme é muito pequena se comparada ao tamanho dos poros da sílica e, conseqüentemente, não chega a produzir variações detectáveis na distribuição de tamanho dos seus poros. Pode-se portanto afirmar, a partir desses resultados, que as três sucessivas reações de enxerto não produziram aglomerados de alumina na superfície da sílica.

A mesma interpretação é obtida quando são analisados os valores das áreas superficiais e dos volumes dos poros dos materiais, que são apresentados na Tabela 2. Pode-se observar que os valores de área superficial e volume dos poros foram semelhantes para todas as amostras. A pequena diminuição da área superficial com o aumento da incorporação de alumina na superfície deve ser considerada, no máximo, como uma leve tendência. Isto porque as diferenças observadas encontram-se na faixa de erro da técnica, que pode alcançar
Tabela 2. Resultados obtidos a partir das isotermas de adsorção e dessorção de $\mathrm{N}_{2}$

\begin{tabular}{lcc}
\hline Amostra & $\mathrm{S} \mathrm{a}^{\mathrm{a}} \mathrm{m}^{2} \mathrm{~g}^{-1 \mathrm{c}}$ & $\mathrm{Vp}^{\mathrm{b}} / \mathrm{cm}^{3} \mathrm{~g}^{-1 \mathrm{c}}$ \\
\hline Sílica pura & 250 & 0,44 \\
$\mathrm{Al}_{2} \mathrm{O}_{3} / \mathrm{SiO}_{2}$ (I) & 237 & 0,47 \\
$\mathrm{Al}_{2} \mathrm{O}_{3} / \mathrm{SiO}_{2}$ (II) & 238 & 0,40 \\
$\mathrm{Al}_{2} \mathrm{O}_{3} / \mathrm{SiO}_{2}$ (III) & 233 & 0,38 \\
\hline
\end{tabular}

${ }^{\mathrm{a}}=$ Área superficial $;{ }^{\mathrm{b}}=$ volume de poros; ${ }^{\mathrm{c}}=$ desvio máximo de $10 \%$.

$10 \%$. Essa tendência para diminuição da área superficial e volume de poros com a incorporação de alumina na matriz sílica pode ser explicada considerando-se a possibilidade de fechamento parcial de microporos $(\phi<2 \mathrm{~nm})^{28}$ que não podem ser detectados a partir das isotermas de adsorção e dessorção de nitrogênio.

Os materiais também foram submetidos à análise por espectroscopia na região do infravermelho. Os espectros relativos à região de estiramento Si-O são apresentados na Figura 4. Pode-se observar claramente uma banda em $c a .970 \mathrm{~cm}^{-1}$, devida à presença de $\mathrm{Si}-\mathrm{OH}{ }^{29}$, que diminui progressivamente com as sucessivas reações de enxerto, isto é, com o aumento da incorporação de óxido de alumínio. No material $\mathrm{Al}_{2} \mathrm{O}_{3} / \mathrm{SiO}_{2}$ (III), a banda de estiramento $\mathrm{Si}-\mathrm{OH}$ praticamente desapareceu.

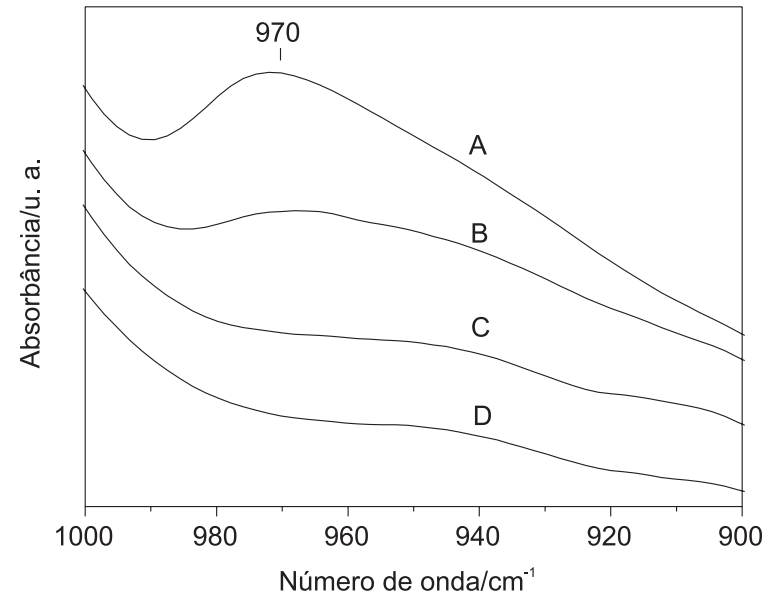

Figura 4. Espectros no infravermelho dos materiais dispersos em $\mathrm{KBr}(0,5 \%)$. A) sílica pura; B) $\mathrm{Al}_{2} \mathrm{O}_{3} / \mathrm{SiO}_{2}(\mathrm{I})$; C) $\mathrm{Al}_{2} \mathrm{O}_{3} / \mathrm{SiO}_{2}$ (II) e D) $\mathrm{Al}_{2} \mathrm{O}_{3} / \mathrm{SiO}_{2}$ (III)

Portanto, nas reações de enxerto de óxido de alumínio sobre sílica, pode-se considerar que o recobrimento progressivo do óxido de alumínio ocorre preferencialmente na superfície de sílica, com tendência de recobrimento completo, na forma de monocamada. Mesmo na amostra $\mathrm{Al}_{2} \mathrm{O}_{3} / \mathrm{SiO}_{2}$ (III), que apresenta maior grau de incorporação de alumínio (Tabela 1), a dispersão do óxido de alumínio é bastante homogênea, visto que não foi observada a formação de aglomerados que pudessem alterar a distribuição de tamanho dos poros.

\section{CONCLUSÕES}

Sucessivas reações de enxerto de óxido de alumínio sobre a superfície de sílica gel resultam em aumento progressivo de incorporação de alumina. O recobrimento do óxido de alumínio é bastante homogêneo sobre a superfície da sílica. A incorporação de até 5,5\% em massa de alumínio ocorre preferencialmente na superfície da sílica, sem formação de aglomerados de alumina, visto 
que não foram detectadas variações na distribuição de tamanho de poros originais da matriz sílica. Portanto, pode-se considerar que o método de enxerto é eficiente para a produção de monocamada de óxido de alumínio disperso sobre a superfície da sílica gel.

\section{AGRADECIMENTOS}

Os autores agradecem ao CNPq, à FAPERGS e COPESUL pelo apoio financeiro e ao CME-UFRGS pelo uso do microscópio eletrônico.

\section{REFERENCIAS}

1. Sica, A. M.; Baibich, I. M.; Gigola, C. E.; J. Mol. Catal. A: Chem. 2002, 195, 225.

2. Sica, A. M.; Dos Santos, J. H. Z.; Baibich, I. M.; Gigola, C. E.; J. Mol. Catal. A: Chem. 1999, 137, 287.

3. Chuah, G. K.; Jaenicke, S.; Liu, S.H.; Hu, X.C.; Appl. Surf. Sci. 2001, $169-$ 170, 253.

4. Monaci, R.; Rombi, E.; Solinas, V.; Sorrentino, A.; Santacesaria, E.; Colon, G.; Appl. Catal., A 2001, 214, 203.

5. Benvenutti, E. V.; Lawislak, L. I.; Polyhedron 1998, 17, 1627.

6. Nunes, C. D.; Valente, A.A.; Pillinger, M.; Rocha, J.; Gonçalves, I. S.; Chem.-A Eur J. 2003, 9, 4380.

7. Krause, J. O.; Lubbad, S.; Nuyken, O.; Buchmeiser, M. R.; Adv. Synth. Catal. 2003, 345, 996.

8. Wang, X. X.; Veyre, L.; Lefebvre, F.; Patarin, J.; Basset, J. M.; Microporous Mesoporous Mater. 2003, 66, 169.

9. Dunphy, D. R.; Singer, S.; Cook, A. W.; Smarsly, B.; Doshi, D. A.; Brinker, C. J.; Langmuir 2003, 19, 10403.
10. Keranen, J.; Guimon, C. A.; Auroux, A.; Iiskola, E.I.; Niinisto, L.; Phys Chem. Chem. Phys. 2003, 5, 5333.

11. Cunha, E. V.; Faccin, F.; Moro, C. C.; Benvenutti, E. V.; Quim. Nova 2002, $25,392$.

12. Lazarin, A. M.; Borgo, C. A.; Gushikem, Y.; Quim. Nova 2002, 25, 499.

13. Seckin, T.; Gultek, A.; J. Appl. Polym. Sci. 2003, 90, 3905.

14. Gushikem, Y.; Rosatto, S. S.; J. Braz. Chem. Soc. 2001, 12, 695.

15. Pessoa, C. A.; Gushikem, Y.; Nakagaki, S.; Electroanal. 2002, 14, 1072.

16. Santacesaria, E.; Sorrentino, A.; Di Serio, M.; Ruggiero, A.; J. Mol. Catal. A: Chem. 2003, 204, 617.

17. Benvenutti, E. V.; Gushikem, Y.; J. Braz. Chem. Soc. 1998, 9, 469.

18. Landau, M. V.; Dafa, E.; Kaliya, M. L.; Sem, T.; Herskowitz, M.; Microporous Mesoporous Mater. 2001, 49, 65.

19. Gao. X.; Wachs, I. E.; J. Catal. 2000, 192, 18.

20. Hoffmann, H. S.; Staudt, P. B.; Costa, T. M. H.; Moro, C. C.; Benvenutti, E. V.; Surf. Interface Anal. 2002, 33, 631.

21. Iengo, P.; Di Serio, M.; Sorrentino, A.; Solinas, V.; Santacesaria, E.; Appl. Catal., A 1998, 167, 85.

22. Brunauer, S.; Emmett, P. H.; Teller, E.; J. Am. Chem. Soc. 1938, 60, 309.

23. Barret, E. P.; Joyner, L. G.; Halenda, P. P.; J. Am. Chem. Soc. 1951, 73, 373.

24. Farrauto, R. J.; Heck, R. M.; Catal. Today 1999, 51, 351.

25. Agrafiotis, C.; Tsetsekou, A.; J. Eur. Ceram. Soc. 2002, 22, 423.

26. Sing, K. S. W.; Everett, D. H.; Haul, R. A. W.; Moscou, L.; Pierotti, R. A.; Rouquérol, J.; Siemieniewska, T.; Pure Appl. Chem. 1985, 57, 603.

27. Gregg, S. J.; Sing, K. S. W.; Adsorption, Surface Area and Porosity, Academic Press: London, 1982, chap. 3, 4.

28. Rouquerol, J.; Avnir, D.; Fairbridge, C. W.; Everett, D. H.; Haynes, J. M. Pernicone, N.; Ramsay, J. D. F.; Sing, K. S. W.; Unger, K. K.; Pure Appl. Chem. 1994, 66, 1739.

29. Fidalgo, A.; Ilharco, L. M.; J. Non-Cryst. Solids 2001, 283, 144. 